\title{
Continuous utility functions on submetrizable hemicompact k-spaces
}

\author{
Alessandro Caterino, Rita Ceppitelli and Francesca Maccarino
}

\begin{abstract}
Some theorems concerning the existence of continuous utility functions for closed preorders on submetrizable hemicompact kspaces are proved. These spaces are precisely the inductive limits of increasing sequences of metric compact subspaces and in general are neither metrizable nor locally compact. These results generalize some well known theorems due to Levin.
\end{abstract}

2000 AMS Classification: 54F05, 91B16.

Keywords: closed preorder, jointly continuous utility function, hemicompact, submetrizable, k-space.

\section{INTRODUCTION}

The problem concerning the existence of a continuous utility function (preorder - preserving map) on a topological space endowed with a (continuous) preorder is called the utility representation problem. In this paper we study the utility representation problem in connection with a not necessarily linear (total) preorder.

Several authors were concerned with non-linear preorders. Indeed, the use of non-linear preorders (or, more generally, non-linear binary relations) may be viewed as more realistic and adequate in order to explain the behavior of an individual. Peleg [27] was the first who presented sufficient conditions for the existence of a continuous utility function for a partial order on a topological space. Peleg solved a problem which was posed by Aumann [1] in the context of expected utility. In particular, Aumann observed that a rational decision-maker may express "indecisiveness" (or equivalently "incomparability") between two alternatives, so that he is not a priori forced to express "indifference" (see also Ok [26]). Later, Mehta [22, 23] followed the spirit of Nachbin [25] in order to show that many general results concerning the continuous utility representation problem can be obtained by combining the classical approach to mathematical 
utility theory with some of the most important results in elementary topology. Herden and Pallack [12] generalized the well known Debreu Theorem concerning the existence of a continuous utility function for a linear preorder on a second countable topological space. In particular, the authors introduced the concept of a weakly continuous preorder on a topological space and in this way provided an appropriate generalization of the notion of a continuous linear preorder. Herden and Pallack results imply the interesting Levin Theorem [16] concerning the existence of a continuous utility function for a not necessarily linear closed preorder on a second countable locally compact Hausdorff space. In connection with the above results, we prove a new generalization of Levin Theorem in the case of closed preorders on spaces not necessarily metrizable or second countable. More precisely, we prove the existence of a continuous utility function for a closed preorder on a submetrizable, hemicompact k-space (submetrizable $k_{\omega}$-space).

An interesting survey on hemicompact k-spaces $\left(k_{\omega}\right.$-spaces) can be found in [11]. Submetrizable $k_{\omega}$-spaces are also studied in $[7,8]$. We recall that submetrizable $k_{\omega}$-spaces are exactly the inductive limits of increasing sequences of metric compact subspaces [8].

In [6] the authors give some representation theorems assuming topological conditions that are very close to the ones of our theorems. They prove that if $X$ is the inclusion inductive limit of a countable chain of compact subspaces $X_{n}$ (that is $X$ is a hemicompact k-space) and $\preceq$ is a preorder on $X$ such that every $\preceq_{\mid X_{n}}$ is closed and order-separable, then $\preceq$ is continuously representable. If we suppose that every $\preceq_{\mid X_{n}}$ is a linear preorder without jumps, this result is equivalent to our result about submetrizable $k_{\omega}$-spaces. In general, the two results are independent.

In the last section we are concerned with the existence of the so-called jointly continuous utility functions. This kind of problems are often considered in mathematical economics. Let $\Gamma$ be a family of preorders (on a topological space $X$ ) endowed with a given topology. The utility representation problem in this context consists in proving the existence of a continuous map $u: \Gamma \times X \longrightarrow \mathbb{R}$ such that $u(\preceq, \cdot): X \longrightarrow \mathbb{R}$ is a utility function for every $\preceq \in \Gamma$. In mathematical economics $X$ and $\Gamma$ can be interpreted as a commodity space and respectively as a space of preference relations of economic agents.

In the second half of the last century, this problem was extensively treated in the literature. A survey of these results can be found in [5]. We quote $[14,9,13,20,4]$ where the existence of jointly continuous functions is proved in the case of linear preorders. Levin in $[16,17,18]$ proved a general theorem for not necessarily linear preorders in second countable locally compact spaces. In the present paper we prove a generalization of Levin results in a nonmetrizable setting. More precisely, we prove the existence of a jointly utility continuous function when $X$ is a submetrizable hemicompact k-space and $\Gamma$ is metrizable or both $\Gamma$ and $X$ are submetrizable hemicompact and the product $\Gamma \times X$ is a $\mathrm{k}$-space. 


\section{Preliminaries}

A reflexive and transitive binary relation on an arbitrary set $X$ is called a preorder $\preceq$. The preorder $\preceq$ on $X$ is linear if $[x \preceq y] \vee[y \preceq x]$ for every $x, y \in X$. An anti-symmetric preorder is said to be an order. If $\preceq$ is a preorder on $X$ the associated asymmetric relation $\prec$ is defined by $[x \prec y \Leftrightarrow(x \preceq y) \wedge \neg(y \preceq x)]$. If $\preceq$ is a preorder on a set $X$, then we will refer to the pair $(X, \preceq)$ as a preordered set.

Let $(X, \preceq)$ be a preordered set. The family of all the sets of the form ]$a,+\infty[=\{x \in X: a \prec x\}$ and $]-\infty, a[=\{x \in X: x \prec a\}$, where $a \in X$, is a subbasis for a topology on $X$. This topology, denoted by $\tau_{\preceq}$ is the order topology induced by $\preceq$ and $\left(X, \tau_{\preceq}\right)$ is called a preordered topological space.

If $(X, \preceq)$ is a preordered set and $\tau$ is a topology on $X$, then the preorder $\preceq$ is said to be closed with respect to $\tau$ if its graph $\{(x, y) \in X \times X: x \preceq y\}$ is a closed subset of $X \times X$. The preorder $\preceq$ is said to be continuous with respect to $\tau$ (or $\tau$-continuous) if for every $a \in X$ the sets $[a,+\infty[=\{x \in X: a \preceq x\}$ and $]-\infty, a]=\{x \in X: x \preceq a\}$ are $\tau$-closed. We recall that if the preorder $\preceq$ is linear, then $\preceq$ is closed iff it is continuous iff $\tau \preceq \subset \tau$.

If $(X, \preceq)$ is a preordered set then a real-valued function $f$ on $X$ is said to be

(i) isotone if for every $x, y \in X,[x \preceq y \Rightarrow f(x) \leq f(y)]$

(ii) preorder-preserving if $f$ is isotone and $[x \prec y \Rightarrow f(x)<f(y)]$.

If $(X, \preceq)$ is a linearly preordered set, then (ii) is equivalent to

(iii) for every $x, y \in X,[x \preceq y \Longleftrightarrow f(x) \leq f(y)]$.

If the preorder $\preceq$ is interpreted as a preference relation on a set $X$ of alternatives then a real-valued preorder-preserving function is also called a utility function or a utility representation of the preorder.

The notions of network and the corresponding netweight seem useful tools in the theory of representation. Spaces with a countable network are a natural generalization of the second countable spaces. A network in a topological space $X$ is a family $\mathcal{N}$ of subsets of $X$ such that every open set of $X$ is union of elements of $\mathcal{N}$. As usual, the net weight of $X$ is defined by

$$
n w(X)=\min \{|\mathcal{N}|: \mathcal{N} \text { is a network for } X\} .
$$

We recall that the netweight is monotone, that is, if $X \subset Y$ then $n w(X) \leq$ $n w(Y)$.

\section{UtiLITY REPRESENTATIONS FOR NON-LINEAR PREORDERS}

We consider the problem of the existence of a continuous utility function on a topological preordered space when the preorder is not necessarily linear. We begin with some interesting results due to Levin. We remark that the techniques used in this case can be very different from those used when $\preceq$ is a linear preorder. Some results on function spaces are sometimes used to solve this kind of problems.

If $X$ is a topological space, let $C(X, \mathbb{R})$ be the set of real continuous functions defined on $X$. We denote by $C_{p}(X, \mathbb{R})$ and $C_{k}(X, \mathbb{R})$ the topological vector 
spaces $\left(C(X, \mathbb{R}), \tau_{p}\right)$ and $\left(C(X, \mathbb{R}), \tau_{k}\right)$, where $\tau_{p}$ is the topology of pointwise convergence and $\tau_{k}$ is the topology of compact convergence.

Theorem 3.1 ([16, Levin]). Let $X$ be a metrizable space such that there exists a sequence $\left\{X_{n}\right\}$ of compact subsets of $X$ with the properties:

(1) $X=\bigcup_{n=1}^{\infty} X_{n}$;

(2) a real-valued function $f$ on $X$ is continuous if and only if its restriction to each $X_{n}$ is continuous.

Then for every closed preorder $\preceq$ there is a continuous utility function on $X$.

Note that if $X$ is a second countable locally compact Hausdorff space, then $X$ satisfies the hypotheses of Theorem 3.1.

Now, the next result extends the above Levin Theorem to non-metrizable case. We need some definitions and a lemma that is a well known result on hemicompact k-spaces.

A topological space $X$ is said to be hemicompact if there is a sequence $\left\{K_{n}\right\}$ of compact subsets of $X$ which is cofinal in the set of all compact subsets of $X$, that is, every compact subset of $X$ is contained in $K_{n}$ for a suitable $n$.

A space $X$ is called a k-space if $A \subset X$ is open if and only if $A \cap K$ is open in $K$ for every compact set $K$ of $X$, that is, $X$ has the weak topology with respect to the family of all compact subsets of $X$.

We recall that the class of the hemicompact k-spaces coincides with the one of the $k_{\omega}$-spaces [11]. A $k_{\omega}$-space is a space with a $k_{\omega}$-decomposition, that is, an increasing sequence $\left\{K_{n}\right\}$ of compact subspaces such that $X=\bigcup_{n=1}^{\infty} K_{n}$ and $X$ has the weak topology with respect to the family $\left\{K_{n}\right\}$.

Lemma 3.2 ([10]). Let $X$ be a hemicompact $k$-space and let $\left\{K_{n}\right\}$ be a sequence of compact subsets of $X$ which is cofinal in the set of all compact subsets of $X$. Then a mapping $f: X \rightarrow Y$ is continuous if and only if $f_{\mid K_{n}}$ is continuous for every $n$.

The following lemma generalizes to hemicompact k-spaces an extension theorem ([16], Lemma 2) proved by Levin for compact spaces.

Lemma 3.3. Let $X$ be a hemicompact $k$-space with a closed preorder $\preceq$, let $S$ be a compact subset of $X$ and let $u: S \rightarrow[0,1]$ be a continuous isotone mapping. Then there is a continuous isotone function $f: X \rightarrow[0,1]$ that extends $u$.

Proof. Let $X=\bigcup_{n=1}^{\infty} X_{n}$ where $X_{n} \subset X$ is compact, $X_{n} \subset X_{n+1}$ for every $n \in \mathbb{N}$ and $\left\{X_{n}\right\}$ is cofinal in the set of all compact subsets of $X$. Since $S$ is compact, $S \subset X_{\bar{n}}$ for some $\bar{n} \in \mathbb{N}$. By Lemma 2 in [16], the function $u$ can be extended to $X_{\bar{n}}$. Using a recursive process and applying Lemma 3.2, the function $u$ can be continuously extended to all of $X$. 
We recall that a topological space $X$ is said to be submetrizable if there exists a coarser metrizable topology on $X$.

Theorem 3.4. Let $X$ be a submetrizable space such that there exists a sequence $\left\{X_{n}\right\}$ of compact subsets of $X$ with the properties:

(1) $X=\bigcup_{n=1}^{\infty} X_{n}$;

(2) a real-valued function $f$ on $X$ is continuous if and only if its restriction to each $X_{n}$ is continuous.

Then for every closed preorder $\preceq$ there is a continuous utility function on $X$.

Proof. It is not restrictive to suppose that $X_{n} \subset X_{n+1}$, for every $n \in \mathbb{N}$. As in the proof of Theorem 2 in [5], if

$$
H=\{u \in C(X, \mathbb{R}): x \preceq y \Longrightarrow u(x) \leq u(y)\}
$$

is the cone of real continuous isotone mappings defined on $X$, then $x \preceq y$ if and only if $u(x) \leq u(y)$ for every $u \in H$. The hypothesis 1 ) and the submetrizability of $X$ imply that $X$ has a countable network. In fact, every compact submetrizable $X_{n}$ is metrizable and second countable. Therefore if $\mathcal{B}_{n}=\left\{B_{i}^{n}\right\}_{i \in \mathbb{N}}$ is a countable base of the (metrizable) subspace $X_{n}$, then $\mathcal{B}=\bigcup \mathcal{B}_{n}$ is a countable network of $X .\left(C(X, \mathbb{R}), \tau_{p}\right)$ has a countable network, too (see [21]). Since the netweight is monotone, we deduce that also $\left(H, \tau_{p}\right)$ has a countable network and so it is separable. Let $\left\{u_{n}\right\}_{n \in \mathbb{N}}$ be a $\tau_{p}$-dense sequence in $H$.

Now, it is possible to show that $x \preceq y$ if and only if $u_{n}(x) \leq u_{n}(y)$ for every $n \in \mathbb{N}$. On the contrary, let $x, y \in X$ such that $u_{n}(x) \leq u_{n}(y)$ for every $n \in \mathbb{N}$ but $\neg(x \preceq y)$. Then, using Lemma 3.3, the function $u:\{x, y\} \rightarrow \mathbb{R}$ defined by $u(y)=0, u(x)=1$ can be extended to a function $\tilde{u} \in H$. Let

$$
J_{\tilde{u}}=\left\{g \in C(X, \mathbb{R}):|g(x)-\tilde{u}(x)|<\frac{1}{4},|g(y)-\tilde{u}(y)|<\frac{1}{4}\right\} .
$$

Since $g(x)-g(y)>\frac{1}{2}$ for every $g \in J_{\tilde{u}}$, the density of $\left\{u_{n}\right\}_{n \in \mathbb{N}}$ in $H$ gets a contradiction.

Finally, as in Levin's proof, the function $u: X \rightarrow \mathbb{R}$ defined by

is the desired continuous utility function.

$$
u(x)=\sum_{n=1}^{\infty} 2^{-n} \frac{u_{n}(x)}{1+\left|u_{n}(x)\right|}
$$

The next result is a direct consequence of Theorem 3.4.

Theorem 3.5. Let $X$ be a submetrizable hemicompact $k$-space and let $\preceq$ be a closed preorder on $X$. Then there is a continuous utility function on $X$.

We recall that every first countable hemicompact space is locally compact and second countable (hence metrizable). But, there are submetrizable hemicompact k-spaces that are not metrizable spaces. 
Example 3.6. Let $\mathbb{Z}$ be the set of integers and $\mathbb{R}$ be the set of all real numbers with the usual topology. Consider the quotient space $Y=\mathbb{R} / \mathbb{Z}$ obtained by identifying the set $\mathbb{Z}$ to the point $y_{0} \in Y$. Then $Y$ is a k-space by definition; it cannot be metrizable since it isn't even first countable ( $y_{0}$ fails to have a countable base of neighbourhoods). Moreover, $Y$ is submetrizable and hemicompact.

Example 3.7. The space $S^{\prime}$ of tempered distributions ([7], Example 3.3) is another example of a submetrizable hemicompact k-space that is not metrizable.

In [6] the authors prove some representation theorems assuming topological conditions that are very close to the assumptions of our Theorems 3.4 and 3.5. In [6], Theorem 2, they prove that if $X=\lim _{\subset} X_{n}$ (inclusion inductive limit) where $\left\{X_{n}\right\}$ is a countable chain of compact subspaces of $X$ and $\preceq$ is a preorder on $X$ such that every $\preceq_{\mid X_{n}}$ is closed and order-separable, then $\preceq$ is continuously representable.

We note that a space $X$ is the inclusion inductive limit of a countable chain of compact subspaces if and only if it is a hemicompact k-space. In fact, every inductive limit of a countable family of compact spaces is a $\sigma$-compact $\mathrm{k}$-space since it is a quotient space of the free union $S=\sum X_{n}$ that is $\sigma$-compact and locally compact. The hemicompactness follows by a theorem of Steenrod in [28]. Conversely, it is easy to prove that every hemicompact k-space is the inclusion inductive limit of a countable chain of compact subspaces.

If we suppose that $\preceq_{\mid X_{n}}$ is a linear preorder without jumps for every $n$, our Theorem 3.5 and Theorem 2 in [6] are equivalent. In fact, in this case, since $X_{n}$ is compact then $\tau_{\preceq_{1 X_{n}}}$ coincides with the topology of the subspace $X_{n}$. Then we get $\preceq_{\mid X_{n}}$ is order-separable if and only if $X_{n}$ is metrizable. Further, an inclusion inductive limit of a countable chain $\left\{X_{n}\right\}$ of compact spaces is submetrizable if and only if $X_{n}$ is metrizable for every $n$ (see $[7,8]$ ).

In general, our Theorem 3.5 and Theorem 2 in [6] are independent.

\section{JoINTLY CONTINUOUS UTILITY FUNCTIONS}

Let $X$ be a topological space and let $\Gamma$ be a family of preorders on $X$, endowed with a given topology.

The problem of the existence of jointly continuous functions consists in proving the existence of a continuous map $u: \Gamma \times X \longrightarrow \mathbb{R}$ such that $u(\preceq, \cdot): X \longrightarrow \mathbb{R}$ is a utility function for every $\preceq \in \Gamma$.

In [16] Levin, using a continuous selection theorem of Michael [24], solved the problem when $\Gamma$ is metrizable and $X$ is locally compact and second countable. In the special case in which $\Gamma$ and $X$ are both locally compact and second countable, Levin proved the result without the use of Michael's Theorem.

We begin to extend this result to the case when $\Gamma$ and $X$ are submetrizable and hemicompact and $\Gamma \times X$ is a k-space. Later (Theorem 4.2) we will extend the Theorem of Levin to the case when $X$ is submetrizable and hemicompact, 
$\Gamma$ is metrizable and $\Gamma \times X$ is a k-space. We note that these two results are independent of each other.

Theorem 4.1. Let $\Gamma$ and $X$ be submetrizable hemicompact spaces and suppose $\Gamma \times X$ is a $k$-space. Moreover, assume that the set

$$
G=\{(\preceq, x, y): x \preceq y\}
$$

is closed in $\Gamma \times X \times X$.

Then there exists a continuous function $u: \Gamma \times X \rightarrow[0,1]$ such that, for each $\preceq \in \Gamma, u(\preceq, \cdot)$ is a continuous utility function on $X$.

Proof. As in Section 5 of [16], the preorder $\leq$ on $\Gamma \times X$, defined by $\left(\preceq_{1}, x_{1}\right) \leq\left(\preceq_{2}, x_{2}\right)$ if and only if $\preceq_{1}=\preceq_{2}$ and $x_{1} \preceq_{1} x_{2}$, is closed. Hence $(\Gamma \times X, \leq)$ satisfies all the conditions of Theorem 3.5 and so there is a continuous utility function $u$ on $\Gamma \times X$ with respect to $\leq$. The conclusion follows from the definition of the preorder $\leq$.

Theorem 4.2. Let $\Gamma$ be a metrizable space, let $X$ be a submetrizable hemicompact space and suppose $\Gamma \times X$ is a $k$-space. Moreover, assume that the set

$$
G=\{(\preceq, x, y): x \preceq y\}
$$

is closed in $\Gamma \times X \times X$.

Then there exists a continuous function $u: \Gamma \times X \rightarrow[0,1]$ such that, for each $\preceq \in \Gamma, \quad u(\preceq, \cdot)$ is a continuous utility function on $X$.

Proof. Let $\left\{K_{n}\right\}$ be a sequence of compact subsets of $X$ which is cofinal in the set of all compact subsets of $X$. Then the topology $\tau_{k}$ on $C_{k}(X, \mathbb{R})$ is generated by the countable family of seminorms $p_{n}(f)=\sup _{x \in K_{n}}|f(x)|$. Therefore, the locally convex topological vector space $C_{k}(X, \mathbb{R})$ is metrizable. Moreover, since $X$ is a k-space then $C_{k}(X, \mathbb{R})$ is also complete ([15], Theorem 12) and hence it is a Fréchet space. As in the proof of Theorem 3.4, the hypotheses of hemicompactness and submetrizability imply that $X$ has a countable network and so $C_{p}(X, \mathbb{R})$ is separable. Then $C_{k}(X, \mathbb{R})$ is separable too ([21], Cor. 4.2.2). Finally, by the hypotheses that $\Gamma \times X$ is a k-space, using the same arguments as in Theorem 1 in [16], it is possible to construct the desired jointly continuous utility function $u$.

Acknowledgements. The authors would like to thank Prof. G. Bosi for his appreciated suggestions and comments. 


\section{REFERENCES}

[1] R. Aumann, Utility theory without the completeness axiom, Econometrica 30 (1962), 445-462.

[2] K. Back, Concepts of similarity for utility functions, Journal of Mathematical Economics 15 (1986), 129-142.

[3] G. Beer, Topologies on Closed and Closed Convex Sets, Kluwer (1993).

[4] D. S. Bridges, Preference and utility: a constructive development, Journal of Mathematical Economics 9 (1982), 165-185.

[5] D. S. Bridges and G. B. Mehta, Representations of preference orderings, Springer-Verlag, Berlin, 1995.

[6] J. C. Candeal, E. Indurain and G. B. Mehta, Some utility theorems on inductive limits of preordered topological spaces, Bull. Austral. Math. Soc. 52 (1995), 235-246.

[7] C. Castaing, P. Raynaud de Fitte and A. Salvadori, Some variational convergence results with applications to evolution inclusions, Adv. Math. Econ. 8 (2006), 33-73.

[8] C. Castaing, P. Raynaud de Fitte and M. Valadier, Young Measures on Topological Spaces with Applications in Control Theory and Probability Theory, Kluwer Academic Publishers Dordrecht 571 (2004).

[9] G. Debreu, Neighbouring economic agents, La Décision. Paris: Editions du Centre National de la Recherche Scientifique (1969).

[10] R. Engelking, General Topology, Polish Scientific Publishers, Warszaw, (1977).

[11] S. P. Franklin and B. V. Smith Thomas, A survey of $k_{\omega}$-spaces, Topology Proceedings 2 (1977), 111-124.

[12] G. Herden and A. Pallack, On the continuous analogue of the Szpilrajn Theorem I, Mathematical Social Sciences 43 (2002), 115-134.

[13] W. Hildebrand, On economies with many agents, Journal of Economic Theory 2 (1970), $1-188$.

[14] Y. Kannai, Continuous properties of the core of a market, Econometrica 38 (1970), 791-815.

[15] J. L . Kelley, General topology, D. Van Nostrand Company (1955).

[16] V. L. Levin, A continuous utility theorem for closed preorders on a $\sigma$-compact metrizable space, Soviet. Math. Dokl. 28 (1983), 715-718.

[17] V. L. Levin, Measurable utility theorems for closed and lexicographic preference relations, Soviet. Math. Dokl. 27 (1983), 639-643.

[18] V. L. Levin, Functionally closed preorders and strong stochastic dominance, Soviet. Math. Dokl. 32 (1985), 22-26.

[19] V. L. Levin and A. A. Milyutin, The problem of mass transfer with a discontinous cost function and a mass statement of the duality problem for convex extremal problems, Russian Math. Surveys 34 (1978), no. 3, 1-78.

[20] A. Mas-Colell, On the continuous representation of preorders, International Economic Review, 18 (1977), 509-513.

[21] R. A. McCoy and I. Ntantu, Topological properties of spaces of continuous functions, Springer-Verlag (1980).

[22] G. B. Mehta, Some general theorems on the existence of order-preserving functions, Mathematical Social Sciences 15 (1988), 135-146.

[23] G. B. Mehta, Preference and Utility, in Handbook of Utility Theory, Volume 1, eds. S. Barberà, P. Hammond and C. Seidl. Dordrecht: Kluwer Academic Publishers, (1998), $1-47$.

[24] E. Michael, Continous selections, Annals of Mathematics 63 (1956), 361-382.

[25] L. Nachbin, Topology and order, Van Nostrand, Princeton (1965).

[26] E. A. Ok, Utility representation of an incomplete preference relation, Journal of Economic Theory 104 (2002), 429-449.

[27] B. Peleg, Utility functions for partially ordered topological spaces, Econometrica, 38 (1970), 93-96. 
[28] N. E. Steenrod, A convenient category of topological spaces, Mich. Math. J. 14 (1967), $133-152$.

ReCEIVED November 2008

AcCePted November 2009

Alessandro Caterino (caterino@dmi.unipg.it)

Dipartimento di Matematica e Informatica, Università degli Studi di Perugia, Via Vanvitelli 1, 06123, Perugia, Italy (corresponding author)

Rita Ceppitelli (matagria@dmi.unipg.it)

Dipartimento di Matematica e Informatica, Università degli Studi di Perugia, Via Vanvitelli 1, 06123, Perugia, Italy

Francesca MaCCARINO (maccarinofrancesca@libero.it) Via Pian del Vantaggio 10A, 05018 Orvieto (Terni), Italy 